\title{
Observation of Lymphangioma of the Duodenum by a Magnifying Endoscope with a Narrow-Band Imaging System
}

\author{
Masaya Iwamuro ${ }^{a, b}$ Yoshinari Kawai ${ }^{a} \quad$ Katsuyoshi Takata ${ }^{c}$ \\ Hiroyuki Okada $^{d}$ Kazuhide Yamamoto ${ }^{b}$ \\ ${ }^{a}$ Department of Gastroenterology, Onomichi Municipal Hospital, Onomichi; \\ Departments of ${ }^{b}$ Gastroenterology and Hepatology and ${ }^{C}$ Pathology, Okayama \\ University Graduate School of Medicine, Dentistry and Pharmaceutical Sciences, \\ and ${ }^{\mathrm{d}}$ Department of Endoscopy, Okayama University Hospital, Okayama, Japan
}

\section{Key Words}

Lymphangioma $\cdot$ Duodenal neoplasms $\cdot$ Magnifying endoscope

\begin{abstract}
Among duodenal tumors, lymphangioma is relatively infrequent. In this case report, we describe the case of a 65-year-old Japanese man with duodenal lymphangioma diagnosed by esophagogastroduodenoscopy. Endoscopically, the tumor appeared as a soft submucosal tumor with white spots. When the white spots were grasped by biopsy forceps, milky liquid exuded from the tumor. Additionally, observation by a magnifying endoscope with narrowband imaging revealed elongated microvessels on the surface. We speculated that this feature was formed because the duodenal villi were dilated and the microvessels were stretched due to the retention of chyle. These endoscopic findings are key features in the diagnosis of duodenal lymphangioma.
\end{abstract}

\section{Introduction}

Lymphangioma is generally regarded as a malformation of segregated lymphatic tissue that has failed to communicate with the normal lymphatic system. Most lymphangiomas arise in the neck and axilla; the gastrointestinal tract is relatively infrequently affected [1-3]. Although the macroscopic features of duodenal lymphangioma are known as submucosal tumors with white-colored spots [4-8], magnifying endoscopic images have never been re- 
ported. In this paper, we describe a case of duodenal lymphangioma observed by a magnifying endoscope with a narrow-band imaging system. Macroscopically, the duodenal lesion appeared as a submucosal tumor with white spots as in the previously reported cases. It was noteworthy that a milky fluid flowed into the duodenal lumen when the tumor was grasped by biopsy forceps. Additionally, observation by a magnifying endoscope with narrow-band imaging revealed elongated microvessels on the surface of the white spots. Based on these endoscopic features, duodenal lymphangioma was highly suspected and the diagnosis was histopathologically confirmed by biopsy examination. The endoscopic features of duodenal lymphangioma are discussed.

\section{Case Presentation}

A 65-year-old Japanese man presented to Onomichi Municipal Hospital with anorexia and abdominal fullness lasting for 1 week. He had been taking no medications. He had no particular past history except for appendicitis. His blood pressure was 127/80 $\mathrm{mm} \mathrm{Hg}$, and his body temperature was $36.6^{\circ} \mathrm{C}$. Physical examination revealed mild tenderness around the umbilicus, but there was no evidence of peritoneal irritation sign. His white blood count was elevated to $11,900 / \mu \mathrm{l}$ and blood urea nitrogen was $52.2 \mathrm{mg} / \mathrm{dl}$. Other laboratory findings were within normal ranges.

Esophagogastroduodenoscopy revealed an active peptic ulceration in the angular portion of the stomach, which was considered the cause of his symptoms. No cancer cells were detected in the biopsy specimen from the gastric ulcer. Additionally, a soft submucosal tumor approximately $20 \mathrm{~mm}$ in diameter, the top of which showed white spots, was demonstrated in the duodenal second portion (fig. 1a). Narrow-band imaging (fig. 1b) and indigo carmine spraying (fig. 1c) emphasized the white spots. When the tumor was grasped by biopsy forceps, chyle flowed into the duodenal lumen (fig. 1d). The view by the magnifying endoscope showed elongated microvessels on the surface of the white spots (fig. 2). Based on these features, duodenal lymphangioma was highly suspected. Subsequent histopathological examination of the biopsy specimen revealed dilated lymphatic structures in the duodenal mucosa and thereby confirmed the diagnosis of duodenal lymphangioma (fig. 3). The patient's symptoms were relieved after the initiation of antacids for his active gastric ulceration. Additionally, Helicobacter pylori bacteria were eradicated, as shown by a positive rapid urease test result. No particular treatment was applied to the duodenal lymphangioma.

\section{Discussion}

Duodenal lymphangioma is a relatively rare disorder. Terada [9] reported to have found only 2 patients among the 567 patients $(0.35 \%)$ with duodenal benign lesions who underwent endoscopic biopsy. The origin of lymphangiomas is generally regarded as obstruction or dysembryoplasia of lymphatic tissue that lacks communication with the systemic lymphatic system [10]. Pathologically, it is characterized by dilated lymphatics in the submucosa. Lymphangiomas are classically categorized into three types based on pathological findings: capillary, cavernous and cystic lymphangioma. In the present case, a cystic multiple dilated lymphatic structure was observed in the biopsy sample. Therefore, the patient was diagnosed with cystic lymphangioma of the duodenum.

Macroscopically, duodenal lymphangiomas appear as submucosal tumors [4-8]. Whitecolored spots on the surface have been reported in previous case reports [4-6] as well as in 
our case. In the present case, white liquid material flowed from the tumor when the white spots were grasped by biopsy forceps. Exudation of milky fluid from duodenal lymphangioma has also been reported by other authors $[4,7]$. Consequently, we speculate that the chyle retained in the dilated lymphatics is endoscopically seen as white spots. In our patient, flat white spots were visualized by a magnifying endoscope (fig. 2a). Additionally, narrow-band imaging view emphasized elongated microvessels on the surface of the white spots. To the best of our knowledge, this is the first report describing the findings of duodenal lymphangioma by a magnifying endoscope. We consider that the features observed in our patient were formed because the duodenal villi were dilated and the microvessels were stretched due to the retention of chyle.

As described above, lymphangioma is generally regarded as a benign tumor and rarely requires specific treatment, unless it is accompanied by infection, fistula or hemorrhage [6]. Rapid tumor growth can be seen in rare instances as a result of increasing lymphatic flow and sudden closure of drainage channels due to infection or other inflammatory processes [5]. Invasion to the surrounding tissues via endothelial budding or sprouting, without malignant features, has also been noted in previous case reports [11,12]. In the present patient, no treatment was initiated for duodenal lymphangioma because the patient had none of these complications.

In conclusion, we describe a case of duodenal lymphangioma. A soft submucosal tumor with white spots and exudation of chyle was observed by esophagogastroduodenoscopy. Moreover, the view by a magnifying endoscope with narrow-band imaging visualized elongated microvessels. Though sensitivity and specificity must be investigated, we consider that these findings are key features for endoscopic diagnosis of duodenal lymphangioma.

\section{Author Contributions}

M. Iwamuro: manuscript drafting and revision. Y. Kawai and H. Okada: clinical data interpretation and critical revision. K. Takata: pathological diagnosis. K. Yamamoto: approval of the final version of the manuscript.

\section{Disclosure Statement}

No conflicts of interest exist for any of the authors.

\section{References}

1 Enzinger FM, Weiss SW: Tumors of lymph vessels; in Gay SM (ed): Soft Tissue Tumors. St. Louis, Mosby, 1988, pp 614-637.

-2 Breidahl WH, Mendelson RM: Retroperitoneal lymphangioma. Australas Radiol 1995;39:187-191.

-3 Galifer RB, Pous JG, Juskiewenski S, Pasquie M, Gaubert J: Intra-abdominal cystic lymphangiomas in childhood. Prog Pediatr Surg 1978;11:173-238.

4 Elliott RL, Williams RD, Bayles D, Griffin J: Lymphangioma of the duodenum: case report with light and electron microscopic observation. Ann Surg 1966;163:86-92.

5 Fujishiro M, Kamoshida T, Hotra S, Hirai S, Oka Y, Sato M, Okumura M, Inadome Y, Takahashi A: Retroperitoneal lymphangioma with a duodenal lesion in an adult. J Gastroenterol 2002;37:381-386.

6 Fang YF, Qiu LF, Du Y, Jiang ZN, Gao M: Small intestinal hemolymphangioma with bleeding: a case report. World J Gastroenterol 2012;18:2145-2146.

7 Sriram PV, Weise C, Seitz U, Brand B, Schroder S, Soehendra N: Lymphangioma of the major duodenal papilla presenting as acute pancreatitis: treatment by endoscopic snare papillectomy. Gastrointest Endosc 2000;51:733-736. 
Iwamuro et al:: Observation of Lymphangioma of the Duodenum by a Magnifying Endoscope with a Narrow-Band Imaging System

8 Ikura Y, Hashimoto T, Takamine Y, Tani T, Konishi Y, Uchida H, Shirane H, Kajiwara T: Lymphangioma of the duodenum: report of a case. Surg Today 1994;24:160-163.

$>9$ Terada T: Pathologic observations of the duodenum in 615 consecutive duodenal specimens: I. Benign lesions. Int J Clin Exp Pathol 2012;5:46-51.

10 Balderramo DC, Di Tada C, de Ditter AB, Mondino JC: Hemolymphangioma of the pancreas: case report and review of the literature. Pancreas 2003;27:197-199.

11 Nuzzo G, Lemmo G, Marrocco-Trischitta MM, Boldrini G, Giovannini I: Retroperitoneal cystic lymphangioma. J Surg Oncol 1996;61:234-237.

12 Raszkowski HJ, Rehbock DJ, Cooper FG: Mesenteric and retroperitoneal lymphangioma. Am J Surg 1959;97: 363-367.
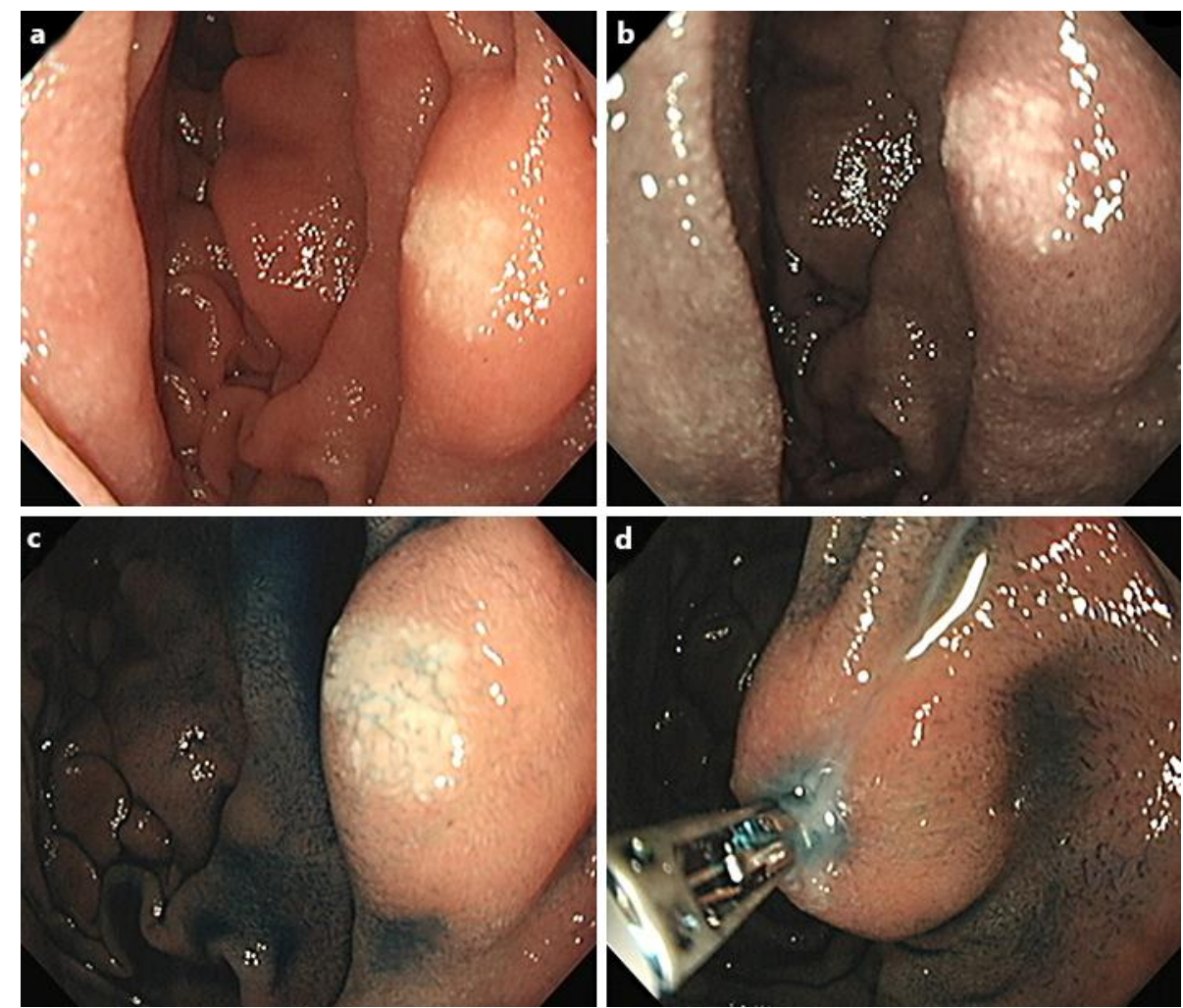

Fig. 1. Endoscopic images of the duodenal second portion. a A soft submucosal tumor with white spots was seen. The white spots were emphasized by narrow-band imaging (b) and indigo carmine dye spraying (c). $\mathbf{d}$ When the white spots were grasped by biopsy forceps, milky fluid flowed into the duodenal lumen. 


\begin{tabular}{ll|l} 
Case Reports in & \multicolumn{2}{l}{} \\
\cline { 2 - 3 } Gastroenterology & Case Rep Gastroenterol 2013;7:229-233 \\
\cline { 2 - 3 } & DOI: $10.1159 / 000351831$ & $\begin{array}{l}\text { C 2013 S. Karger AG, Basel } \\
\text { www.karger.com/crg }\end{array}$ \\
\cline { 2 - 3 } & Iwamuro et al: Observation of Lymphangioma of the Duodenum by a Magnifying
\end{tabular}

Iwamuro et a : Observation of Lymphangioma of the Duodenum by a Magnifying

Endoscope with a Narrow-Band Imaging System

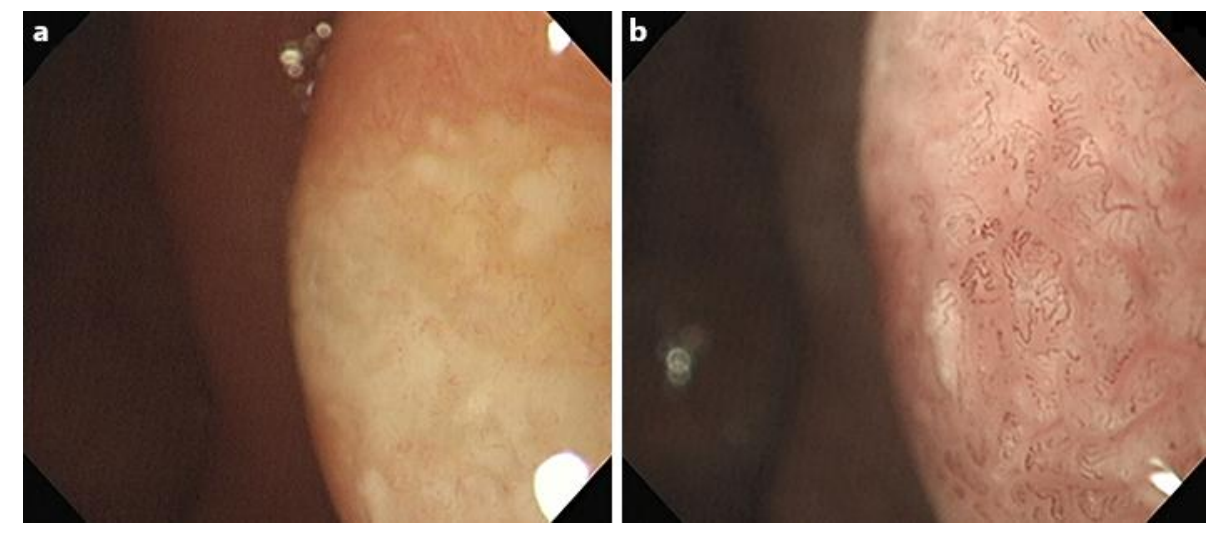

Fig. 2. Magnifying endoscopic images of the tumor. a Flat white spots and microvessels on the surface were seen. $\mathbf{b}$ The elongated microvessels were more clearly visualized by narrow-band imaging.
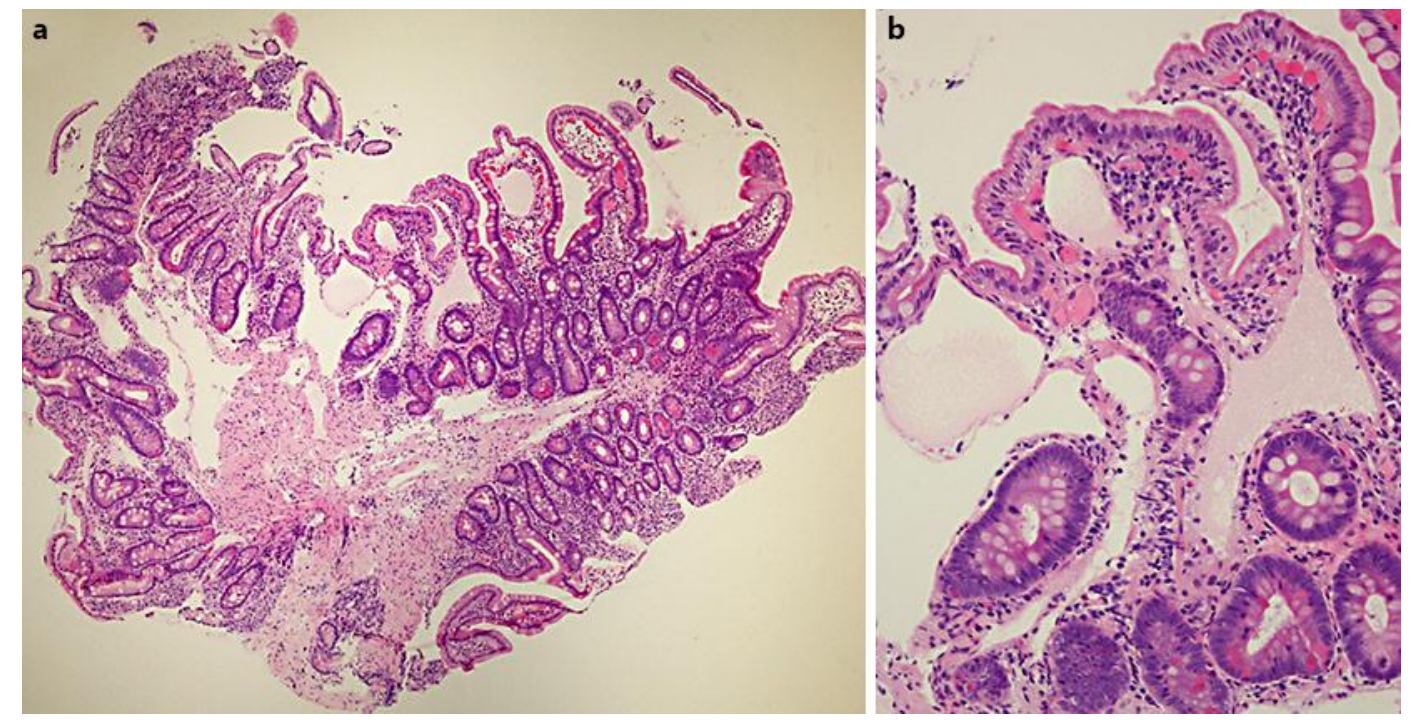

Fig. 3. Pathological images of the duodenal tumor. Multiple dilated lymphatic structures were detected in the biopsy specimen. The diagnosis of duodenal lymphangioma was thus confirmed. Hematoxylin and eosin staining, original magnification $\times 10$ (a) and $\times 20$ (b). 